\title{
Charmed Mesons and Charmonia: Three-Meson Strong Couplings
}

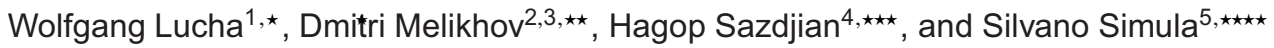 \\ ${ }^{1}$ Institute for High Energy Physics, Austrian Academy of Sciences, Nikolsdorfergasse 18, A-1050 Vienna, \\ Austria \\ ${ }^{2}$ D. V. Skobeltsyn Institute of Nuclear Physics, M. V. Lomonosov Moscow State University, 119991, Moscow, \\ Russia \\ ${ }^{3}$ Faculty of Physics, University of Vienna, Boltzmanngasse 5, A-1090 Vienna, Austria \\ ${ }^{4}$ IPN, CNRS/IN2P3, Universitè Paris-Sud 11, F-91406 Orsay, France \\ ${ }^{5}$ INFN, Sezione di Roma Tre, Via della Vasca Navale 84, I-00146 Roma, Italy
}

\begin{abstract}
Revisiting the strong couplings of three mesons, each of which involves at least one charm quark, proves clear disaccord between quark-model and QCD sum-rule results.
\end{abstract}

\section{Three-meson strong couplings from relativistic constituent-quark model}

We extract the strong couplings of three mesons among which there is, at least, one of the charmonia $\eta_{c}$ and $J / \psi$ from the residues of poles in adequate transition form factors for timelike momentum transfer, which, in turn, are inferred from a relativistic dispersion approach based on a constituent-quark model.

\section{Definitions of strong couplings, transition form factors, decay constants}

Preparatively, let us introduce all the quantities necessary for the formulation of the relation sought, for pseudoscalar mesons $P$, with mass $M_{P}$, and vector mesons $V$, with mass $M_{V}$ and polarization vector $\varepsilon_{\mu}$.

- With momentum transfer $q \equiv p_{1}-p_{2}$, the strong couplings $g_{P P^{\prime} V}$ and $g_{P V^{\prime} V}$ determine the amplitudes

$$
\left\langle P^{\prime}\left(p_{2}\right) V(q) \mid P\left(p_{1}\right)\right\rangle=-\frac{g_{P P^{\prime} V}}{2}\left(p_{1}+p_{2}\right)^{\mu} \varepsilon_{\mu}^{*}(q), \quad\left\langle V^{\prime}\left(p_{2}\right) V(q) \mid P\left(p_{1}\right)\right\rangle=-g_{P V^{\prime} V} \epsilon_{\varepsilon^{*}}(q) \varepsilon^{*}\left(p_{2}\right) p_{1} p_{2} .
$$

- The transition form factors $\mathcal{F}\left(q^{2}\right)=F_{+}^{P>P^{\prime}}\left(q^{2}\right), V^{P>V}\left(q^{2}\right)$ or $A_{0}^{P>V}\left(q^{2}\right)$ enter in the two-meson matrix elements of the vector quark current $j_{\mu} \equiv \bar{q}_{1} \gamma_{\mu} q_{2}$ and the axial-vector quark current $j_{\mu}^{5} \equiv \bar{q}_{1} \gamma_{\mu} \gamma_{5} q_{2}$

$$
\begin{aligned}
& \left\langle P^{\prime}\left(p_{2}\right)\left|j_{\mu}\right| P\left(p_{1}\right)\right\rangle=F_{+}^{P>P^{\prime}}\left(q^{2}\right)\left(p_{1}+p_{2}\right)_{\mu}+\cdots, \quad\left\langle V\left(p_{2}\right)\left|j_{\mu}\right| P\left(p_{1}\right)\right\rangle=\frac{2 V^{P>V}\left(q^{2}\right)}{M_{P}+M_{V}} \epsilon_{\mu \varepsilon^{*}\left(p_{2}\right) p_{1} p_{2}}, \\
& \left\langle V\left(p_{2}\right)\left|j_{\mu}^{5}\right| P\left(p_{1}\right)\right\rangle=\mathrm{i} q_{\mu}\left(\varepsilon^{*}\left(p_{2}\right) p_{1}\right) \frac{2 M_{V}}{q^{2}} A_{0}^{P>V}\left(q^{2}\right)+\cdots .
\end{aligned}
$$

\footnotetext{
^e-mail: Wolfgang.Lucha@oeaw.ac.at

$\star \star$ e-mail: dmitri melikhov@gmx.de

$\star \star \star$ e-mail: sazdjian@ipno.in2p3.fr

$\star \star \star \star$ e-mail: simula@roma3.infn.it
} 
- The vector and pseudoscalar decay constants $f_{V, P}$ govern the meson-vacuum matrix elements of $j_{\mu}^{(5)}$ :

$$
\left\langle 0\left|j_{\mu}\right| V(q)\right\rangle=f_{V} M_{V} \varepsilon_{\mu}(q), \quad\left\langle 0\left|j_{\mu}^{5}\right| P(q)\right\rangle=\mathrm{i} f_{P} q_{\mu} .
$$

In terms of the above quantities, the contributions of the poles residing at the masses $M_{V_{R}}$ or $M_{P_{R}}$ of the relevant vector and pseudoscalar resonances, $V_{R}$ and $P_{R}$, to the form factors introduced in Eqs. (1) read

$$
\begin{aligned}
F_{+}^{P>P^{\prime}}\left(q^{2}\right) & =\frac{g_{P P^{\prime} V_{R}} f_{V_{R}}}{2 M_{V_{R}}} \frac{1}{1-q^{2} / M_{V_{R}}^{2}}+\cdots, \quad V^{P>V}\left(q^{2}\right)=\frac{\left(M_{V}+M_{P}\right) g_{P V V_{R}} f_{V_{R}}}{2 M_{V_{R}}} \frac{1}{1-q^{2} / M_{V_{R}}^{2}}+\cdots, \\
A_{0}^{P>V}\left(q^{2}\right) & =\frac{g_{P P_{R} V} f_{P_{R}}}{2 M_{V}} \frac{1}{1-q^{2} / M_{P_{R}}^{2}}+\cdots .
\end{aligned}
$$

\section{Dispersion formalism relying on relativistic constituent-quark framework}

For the actual theoretical computation of the three transition form factors $\mathcal{F}\left(q^{2}\right)$ lying at the core of our strong-couplings study, past experience leads us to trust in the relativistic constituent-quark model [1]. Adhering to this conviction requires us to match our currents to those built up by constituent quarks, $Q$.

- For heavy-quark currents, this can be easily accomplished by use of corresponding form factors $g_{V, A}$ :

$$
j_{\mu}=g_{V} \bar{Q}_{1} \gamma_{\mu} Q_{2}+\cdots, \quad j_{\mu}^{5}=g_{A} \bar{Q}_{1} \gamma_{\mu} \gamma_{5} Q_{2}+\cdots .
$$

- For light-quark currents, partial axial-current conservation, e.g., renders this rather cumbersome [2]. Following Ref. [3], we use for our model parameter values the constituent-quark masses of Table 1 and

$$
g_{V}=g_{A}=1 .
$$

Table 1. Numerical values of the constituent-quark mass parameters $m_{Q}$ entering in the present investigation [3].

\begin{tabular}{lcccc}
\hline Quark flavour $Q$ & $u$ & $d$ & $s$ & $c$ \\
\hline Quark mass $m_{Q}(\mathrm{GeV})$ & 0.23 & 0.23 & 0.35 & 1.45 \\
\hline
\end{tabular}

Then, by application of the relativistic dispersion formalism, we are put in the position to represent the leptonic decay constants $f_{P, V}$ in the form of dispersion integrals of $\operatorname{spectral}$ densities $\rho_{P, V}(s)$ and the transition form factors $\mathcal{F}\left(q^{2}\right)$ by double dispersion integrals of double spectral densities $\Delta_{\mathcal{F}}\left(s_{1}, s_{2}, q^{2}\right)$,

$$
f_{P, V}=\int \mathrm{d} s \phi_{P, V}(s) \rho_{P, V}(s), \quad \mathcal{F}\left(q^{2}\right)=\int \mathrm{d} s_{1} \phi_{1}\left(s_{1}\right) \int \mathrm{d} s_{2} \phi_{2}\left(s_{2}\right) \Delta_{\mathcal{F}}\left(s_{1}, s_{2}, q^{2}\right),
$$

involving the wave functions of pseudoscalar and vector mesons taking part in the studied reactions [4]

$$
\phi_{P, V}(s)=\frac{\pi}{s^{3 / 4}} \sqrt{\frac{s^{2}-\left(m_{1}^{2}-m^{2}\right)^{2}}{2\left[s-\left(m_{1}-m\right)^{2}\right]}} w_{P, V}\left(\frac{\left(s-m_{1}^{2}-m^{2}\right)^{2}-4 m_{1}^{2} m^{2}}{4 s}\right), \quad \int \mathrm{d} k k^{2} w_{P, V}^{2}\left(k^{2}\right)=1 .
$$

The spectral densities may be derived from one-loop Feynman graphs, of the kind shown in Fig. 1. For the radial meson wave functions $w_{P, V}\left(k^{2}\right)$, it has become customary to assume simple Gaussian shapes:

$$
w_{P, V}\left(k^{2}\right) \propto \exp \left(-\frac{k^{2}}{2 \beta_{P, V}^{2}}\right) .
$$

The necessary input parameter values, drawn from a variety of related sources, are collected in Table 2. 

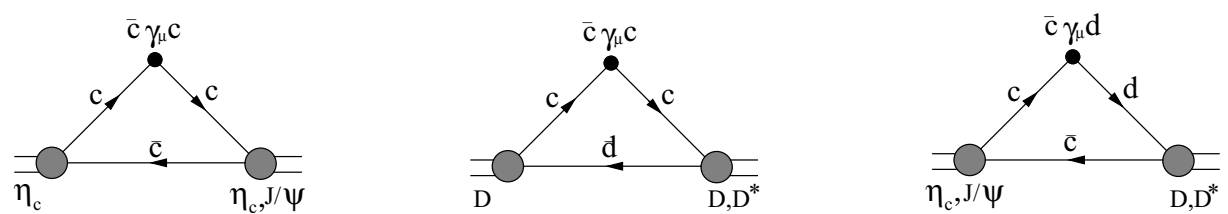

Figure 1. $M_{1}>M_{2}$ meson-meson transitions for $M_{1,2}=\eta_{c}, J / \psi, D, D^{*}$, mediated by the constituent-quark vector currents $\bar{c} \gamma_{\mu} c$ or $\bar{c} \gamma_{\mu} d$ : Feynman graphs yielding the one-loop contributions to the spectral density $\Delta_{\mathcal{F}}\left(s_{1}, s_{2}, q^{2}\right)$.

Table 2. Numerical values of the relevant parameters of the charmed mesons $D_{(s)}^{(*)}$ and the charmonia $\eta_{c}$ and $J / \psi$ : meson mass $M$, leptonic decay constant $f$ defined in Eq. (2), and slope $\beta$ fixing the width of the Gaussian (4) [5].

\begin{tabular}{ccccccc}
\hline Meson & $D$ & $D^{*}$ & $D_{s}$ & $D_{s}^{*}$ & $\eta_{c}$ & $J / \psi$ \\
\hline$M(\mathrm{GeV})$ & 1.87 & 2.010 & 1.97 & 2.11 & 2.980 & 3.097 \\
$f(\mathrm{MeV})$ & $206 \pm 8$ & $260 \pm 10$ & $248 \pm 2.5$ & $311 \pm 9$ & $394.7 \pm 2.4$ & $405 \pm 7$ \\
$\beta(\mathrm{GeV})$ & 0.475 & 0.48 & 0.545 & 0.54 & 0.77 & 0.68 \\
\hline
\end{tabular}

In order to extract the strong couplings under discussion, we determine the momentum dependence for the transition form factors $\mathcal{F}\left(q^{2}\right)$ sufficiently far off their resonances $R$, where $R=V$ for $\mathcal{F}=F_{+}^{P>P^{\prime}}$ and $\mathcal{F}=V^{P>V}$ but $R=P$ for $\mathcal{F}=A_{0}^{P>V}$, interpolate our results by means of the simple parametrization

$$
\mathcal{F}\left(q^{2}\right)=\frac{\mathcal{F}(0)}{\left(1-q^{2} / M_{R}^{2}\right)\left(1-\sigma_{1} q^{2} / M_{R}^{2}+\sigma_{2} q^{4} / M_{R}^{4}\right)}, \quad \operatorname{Res} \mathcal{F}\left(M_{R}^{2}\right)=\frac{\mathcal{F}(0)}{1-\sigma_{1}+\sigma_{2}},
$$

governed by the three parameters $\sigma_{1,2}$ and $\mathcal{F}(0)$, and extrapolate this momentum dependence of $\mathcal{F}\left(q^{2}\right)$ to the resonance region $q^{2} \approx M_{R}^{2}$. From the resulting residues of the meson poles at $q^{2}=M_{R}^{2}$, the strong couplings are found by factorizing off all known quantities such as meson masses and decay constants. In case a particular strong coupling shows up in residues of resonance poles of more than one transition form factor, for such multipresent strong coupling an optimized estimate is obtained by a combined fit.

\section{Strong couplings among three $\eta_{c}$ or $J / \psi$ mesons: $\eta_{c} \eta_{c} J / \psi$ and $\eta_{c} J / \psi J / \psi$}

An illustration of such multipresence is given by the strong coupling $g_{\eta_{c} \eta_{c} \psi}$ [6], with appearance in both - the residue of $F_{+}^{\eta_{c}>\eta_{c}}\left(M_{\psi}^{2}\right)$ arising from the vector current $\bar{c} \gamma_{\mu} c$ coupling, with strength $f_{\psi}$, to $J / \psi$ and

- the residue of $A_{0}^{\eta_{c}>\psi}\left(M_{\eta_{c}}^{2}\right)$ from the axial-vector current $\bar{c} \gamma_{\mu} \gamma_{5} c$ that couples, with strength $f_{\eta_{c}}$, to $\eta_{c}$ :

$$
\operatorname{Res} F_{+}^{\eta_{c}>\eta_{c}}\left(M_{\psi}^{2}\right)=g_{\eta_{c} \eta_{c} \psi} \frac{f_{\psi}}{2 M_{\psi}}, \quad \operatorname{Res} A_{0}^{\eta_{c}>\psi}\left(M_{\eta_{c}}^{2}\right)=g_{\eta_{c} \eta_{c} \psi} \frac{f_{\eta_{c}}}{2 M_{\psi}} .
$$

After such detailed preliminaries, the way how to proceed should be pretty plain: We determine the meson wave-function parameters $\beta_{\eta_{c}, \psi}$ of both charmonia such that the dispersion representation (3) of their decay constants, $f_{\eta_{c}, \psi}$, reproduces the observed values. With these meson vertices at our disposal, we deduce the strong coupling of interest, for each meson-meson transition where this strong coupling enters (for the case of the $\eta_{c} \eta_{c} J / \psi$ coupling, see Fig. 2), from the spectral representation (3) of the form factor corresponding to the respective transition. Thus, our $\eta_{c}$ and $J / \psi P P V$ and $P V V$ couplings are [6]

$$
g_{\eta_{c} \eta_{c} \psi}=25.8 \pm 1.7, \quad g_{\eta_{c} \psi \psi}=(10.6 \pm 1.5) \mathrm{GeV}^{-1}
$$




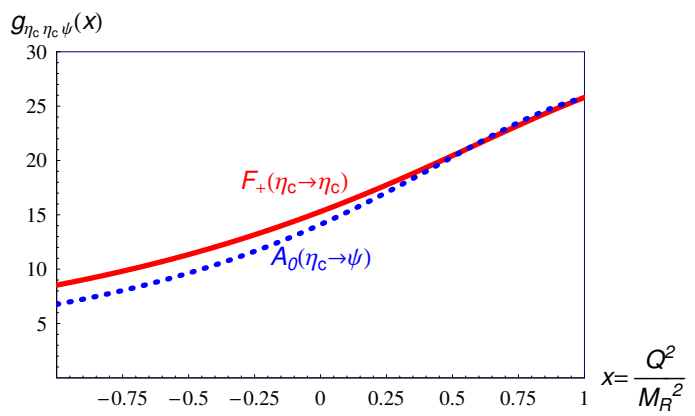

Figure 2. Off-shell strong coupling $g_{\eta_{c} \eta_{c} \psi}(x)$ as function of $x \equiv \frac{q^{2}}{M_{R}^{2}}$ for $\eta_{c}>\eta_{c}$ (red) or $\eta_{c}>J / \psi$ (blue) transitions.

\section{Strong three-meson couplings of the charmonia $J / \psi$ or $\eta_{c}$ to $D_{(s)}$ and $D_{(s)}^{*}$

Along the same route as in Sec. 4 - and by taking into account, in addition, constituent-quark currents involving one $d$ or $s$ quark - we may likewise discuss the strong couplings of $J / \psi$ or $\eta_{c}$ to non-strange $\left(D^{(*)}\right)$ or strange $\left(D_{s}^{(*)}\right)$ charmed mesons. Combining the options sketched in Fig. 3, our findings are [6]

$$
\begin{aligned}
g_{D D \psi} & =26.04 \pm 1.43, & g_{D D^{*} \psi} & =(10.7 \pm 0.4) \mathrm{GeV}^{-1}, \\
g_{D D^{*} \eta_{c}} & =15.51 \pm 0.45, & g_{D^{*} D^{*} \eta_{c}} & =(9.76 \pm 0.32) \mathrm{GeV}^{-1}, \\
g_{D_{s} D_{s} \psi} & =23.83 \pm 0.78, & g_{D_{s} D_{s}^{*} \psi} & =(9.6 \pm 0.8) \mathrm{GeV}^{-1}, \\
g_{D_{s} D_{s}^{*} \eta_{c}} & =14.15 \pm 0.52, & g_{D_{s}^{*} D_{s}^{*} \eta_{c}} & =(8.27 \pm 0.37) \mathrm{GeV}^{-1} .
\end{aligned}
$$

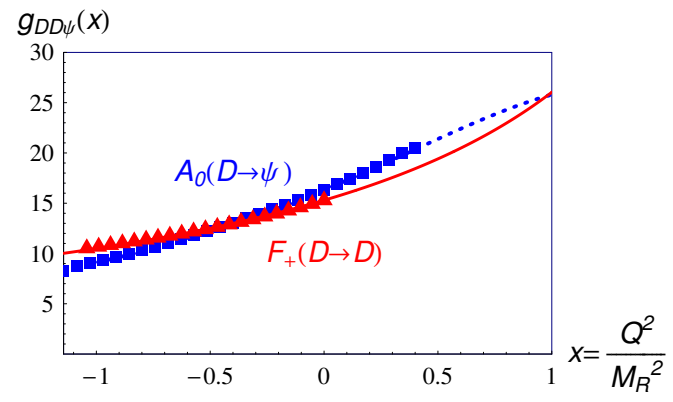

(a)

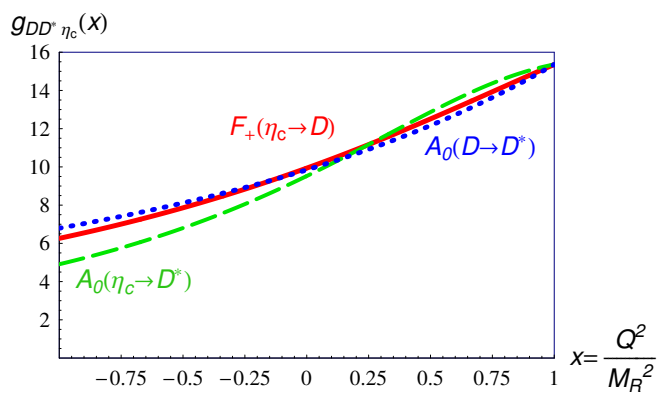

(b)

Figure 3. Off-shell strong couplings $g_{D D \psi}$ and $g_{D D^{*} \eta_{c}}$ to $D^{(*)}$ mesons (with resonances indicated by circumflexes): dependences on $x \equiv \frac{q^{2}}{M_{R}^{2}}$ of (a) $g_{D \hat{D} \psi}(x)=\frac{2 M_{\psi}}{f_{D}}(1-x) A_{0}^{D>\psi}\left(q^{2}\right)$ (blue) and $g_{D D \hat{\psi}}(x)=\frac{2 M_{\psi}}{f_{\psi}}(1-x) F_{+}^{D>D}\left(q^{2}\right)($ red), got with (lines) and without (symbols) interpolation, and of (b) $g_{D D^{*} \eta_{c}}(x)$ (red), $g_{D D^{*} \eta_{c}}(x)$ (blue) and $g_{\hat{D} D^{*} \eta_{c}}(x)$ (green).

\section{Observations, comparison with findings of different origin, conclusions}

Our application of a relativistic dispersion technique, relying on the constituent-quark model, to strong three-meson couplings of quarkonia among each other and to $D_{(s)}^{(*)}$ mesons yields some crucial insights: 
1. The interpolation of our numerical transition-form-factor results found at low $q^{2}$ by means of the ansatz (5) yields values of the resonance-mass fit parameter $M_{R}$ close to the experimental meson masses; this can be interpreted as confirmation of the presence of the poles expected at $q^{2} \approx M_{R}^{2}$.

2. The replacement of the $d$ quark by the $s$ quark (or vice versa) in the quark currents mediating any transition under study enables us to arrive at some estimate of the size of SU(3)-breaking effects. Inspecting Eq. (6), we get a change of the strong couplings under consideration by roughly $10 \%$.

3. Table 3 confronts, for the strong couplings between charmonia and $D_{(s)}^{(*)}$ mesons, the predictions of our dispersive constituent-quark formalism with (available) corresponding figures from QCD sum rules [7-9]; surprisingly, the latter prove to be smaller than our results [6] by a factor of two.

Table 3. Strong couplings of three mesons which involve one $J / \psi$ : findings of the present relativistic constituent quark-model framework [6], confronted with available results [7-9] extracted from the QCD sum-rule approach.

\begin{tabular}{lcccc}
\hline Coupling & $g_{D D \psi}$ & $g_{D D^{*} \psi}\left(\mathrm{GeV}^{-1}\right)$ & $g_{D_{s} D_{s} \psi}$ & $g_{D_{s} D_{s}^{*} \psi}\left(\mathrm{GeV}^{-1}\right)$ \\
\hline Quark model [6] & $26.04 \pm 1.43$ & $10.7 \pm 0.4$ & $23.83 \pm 0.78$ & $9.6 \pm 0.8$ \\
QCD sum rules & $11.6 \pm 1.8[7]$ & $4.0 \pm 0.6[7]$ & $11.96 \pm 1.34[8]$ & $4.30 \pm 1.53[9]$ \\
\hline
\end{tabular}

\section{References}

[1] W. Lucha, F. F. Schöberl, and D. Gromes, Phys. Rep. 200 (1991) 127; F. Cardarelli, E. Pace, G. Salmè, and S. Simula, Phys. Lett. B 357 (1995) 267, arXiv:nucl-th/9507037; R. N. Faustov and V. O. Galkin, Z. Phys. C 66 (1995) 119.

[2] D. Melikhov and B. Stech, Phys. Rev. D 74 (2006) 034022, arXiv:hep-ph/0606203; W. Lucha, D. Melikhov, and S. Simula, Phys. Rev. D 74 (2006) 054004, arXiv:hep-ph/0606281.

[3] D. Melikhov and B. Stech, Phys. Rev. D 62 (2000) 014006, arXiv:hep-ph/0001113.

[4] D. Melikhov, Eur. Phys. J. direct C4 (2002) 2, arXiv:hep-ph/0110087.

[5] C. T. H. Davies et al., Phys. Rev. D 82 (2010) 114504, arXiv:1008.4018 [hep-lat]; W. Lucha, D. Melikhov, and S. Simula, Phys. Lett. B 701 (2011) 82, arXiv:1101.5986 [hep-ph]; D. Bečirević et al., JHEP 1202 (2012) 042, arXiv:1201.4039 [hep-lat]; G. C. Donald et al., Phys. Rev. D 86 (2012) 094501, arXiv:1208.2855 [hep-lat]; W. Lucha, D. Melikhov, and S. Simula, Phys. Lett. B 735 (2014) 12, arXiv:1404.0293 [hep-ph]; K. A. Olive et al. (PDG), Chin. Phys. C 38 (2014) 090001 .

[6] W. Lucha, D. Melikhov, H. Sazdjian, and S. Simula, Phys. Rev. D 93 (2016) 016004, 93 (2016) 019902(E), arXiv:1506.09213 [hep-ph].

[7] R. D. Matheus et al., Int. J. Mod. Phys. E 14 (2005) 555.

[8] B. Osório Rodrigues, M. E. Bracco, and M. Chiapparini, Nucl. Phys. A 929 (2014) 143; arXiv: 1309.1637 [hep-ph].

[9] B. Osório Rodrigues et al., Eur. Phys. J. A 51 (2015) 28; arXiv:1501.03088 [hep-ph]. 